\title{
PMUT-BASED HIGH DATA RATE ULTRASONIC WIRELESS COMMUNICATION LINK FOR INTRA-BODY NETWORKS
}

\author{
Bernard Herrera $^{1}$, Emrecan Demirors ${ }^{1}$, Guofeng Chen ${ }^{1}$, Raffaele Guida ${ }^{1}$, Flavius Pop ${ }^{1}$, Neil Dave ${ }^{1}$, \\ Cristian Cassella ${ }^{1}$, Tommaso Melodia ${ }^{1}$, and Matteo Rinaldi ${ }^{1}$ \\ ${ }^{1}$ Northeastern University, Boston, MA
}

\begin{abstract}
This paper reports on the first demonstration of a high data rate $(0.6 \mathrm{Mbit} / \mathrm{s})$ ultrasonic wireless communication link implemented through Aluminum Nitride (AIN) Piezoelectric Micro Machined Ultrasonic Transducers (PMUTs). Real-time video streaming is demonstrated through a phantom mimicking human tissue, thus proving the feasibility of PMUT-based implantable Body Area Networks (BANs). Two $20 \times 20$ PMUT arrays were used as transceiver elements and an Orthogonal-Frequency-Division-Multiplexing (OFDM) modulation scheme was implemented resulting in a wideband digital transmission link with a data rate of up to $0.6 \mathrm{Mbit} / \mathrm{s}$ at a $5 \mathrm{~cm}$ distance between transmitter and receiver. Channel estimation and Bit-Error-Rate (BER) versus Signal-to-Noise-Ratio (SNR) curves were obtained for both the PMUT link and an implementation based on custom-made and miniaturized ultrasonic bulk Lead Zirconate Titanate (PZT) transducers for comparison. The PMUT implementation, characterized by a wider bandwidth than the PZT transducers, showed two orders of magnitude lower BER for the same SNR as compared to its PZT counterpart, while occupying an approximately 100 times smaller volume $(\sim 3 \mathrm{~mm} \times 3 \mathrm{~mm} \times 0.5 \mathrm{~mm}$ PMUT array vs. $9.5 \mathrm{~mm}$ diameter, $6 \mathrm{~mm}$ thick PZT transducer).
\end{abstract}

\section{INTRODUCTION}

The use of PMUTs in applications such as ultrasound imaging [1], fingerprint scanning [2] and three-dimensional gesture recognition [3] has been widely investigated. Furthermore, several studies focusing on the optimization of specific device performance metrics (such as output pressure or bandwidth) have been reported [4]. However, the application of the PMUT technology for communication purposes remains largely unexplored.

As an example, a high data rate intra-body communication network has potential applications in high resolution wireless endoscopy [5], imaging or biosignal telemetry, continuous ambulatory monitoring of chronic patients, and early disease detection.

The great potential of ultrasonic wireless communication for intra-body networks has been recently demonstrated [6]. Even though a great degree of miniaturization and power consumption optimization was achieved, the implementation is based on Lead Zirconate Titanate (PZT) ultrasonic transducers that remain relatively bulky and are a not fully biocompatible. In fact, the presence of lead in the composition can be toxic for human tissues, particularly in long-term use.

In this article, an ultrasonic transceiver based on miniaturized arrays of AIN PMUTs is demonstrated for the first time, attempting to address these fundamental challenges currently hindering the full deployment of implantable ultrasonic wireless communication devices. AlN is a much more inert material for the intra-body chemistry, which would allow for implants immune to degradation and toxic effects in the long term operation required. Another dramatically enabling feature is that, as the PMUT transducers are built on silicon technology, a single chip implementation of the transducer array and Application-Specific Integrated Circuits

978-1-940470-03-0/HH2018/\$25@2018TRF

DOI 10.31438/trf.hh2018.31
(ASICs) can be envisioned, allowing for drastic miniaturization and power consumption reduction.

Therefore, the achievement of a PMUT-based intra-body communication link perfectly fits the development of an implantable, integrated system offering communication, energy harvesting and sensing capabilities [6]. The use of arrays of PMUTs in an ultrasonic transceiver is also attractive because it enables the implementation of functionalities such as focusing and beamforming that are not attainable with the conventional single-element transducers.

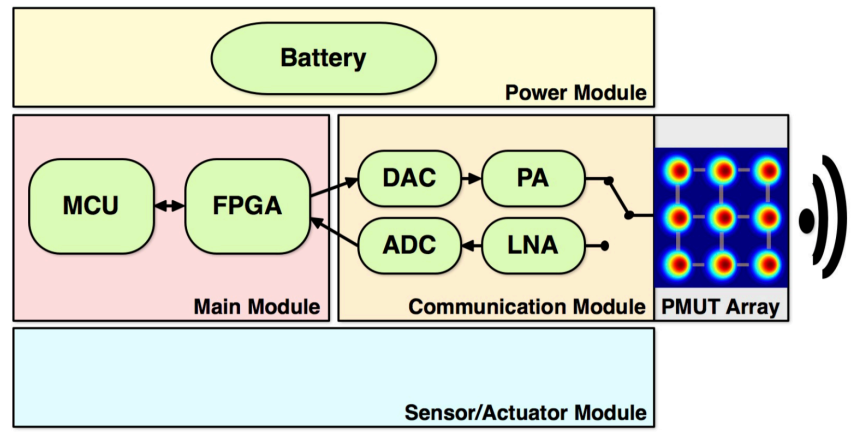

Figure 1: System-wide overview of the application of the PMUT array within an ultrasonic intra-body transceiver.

\section{FABRICATION}

The AlN PMUTs were fabricated on a silicon wafer using a 4 masks microfabrication process. A double-side polished, $300 \mu \mathrm{m}$ silicon wafer was used as a substrate. An $850 \mathrm{~nm}$ silicon dioxide layer was then deposited through Plasma Enhanced Chemical Vapor Deposition (PECVD). A 5nm Pt / 95nm Ti bottom electrode was then formed by electron beam evaporation and patterned through a photo lithography and liftoff process. $800 \mathrm{~nm}$ of the piezoelectric AlN were then reactively sputtered and vias were etched through the layer for access to the bottom electrode by hot phosphoric acid etching. The material stack was completed with a sputtered $5 \mathrm{~nm}$ Ti / 50nm Au top electrode. Back-side alignment photolithography and Deep Reactive Ion Etching (DRIE) were finally used to etch cavities and release the device membrane (fig. 2).
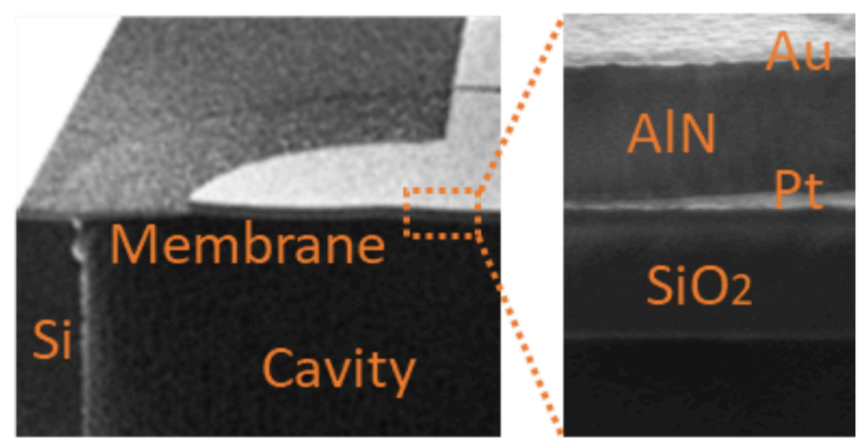

Figure 2: Cross-section SEM images of PMUT element. Left: Overview of the structure: substrate, cavity and membrane. Right: Material stack detail.

Solid-State Sensors, Actuators and Microsystems Workshop Hilton Head Island, South Carolina, June 3-7, 2018 


\section{DEVICE CHARACTERIZATION}

A Digital Holographic Microscope (DHM) was used to characterize the membrane displacement as function of the frequency of the input driving signal. The apparatus is based on laser interferometry; the phase difference information between two perpendicular laser paths, one of which reflects on the sample, is directly related to the displacement of the sample. The measurements were taken for both in-air and water immersed operation by calibrating the length of the laser path.

Operation in air yields a very narrow resonant peak and a higher center frequency. On the other hand, a much wider bandwidth was measured when the PMUTs were operated in water (mimicking conditions of intra-body tissue, Fig. 3), indicating a good coupling to the medium.

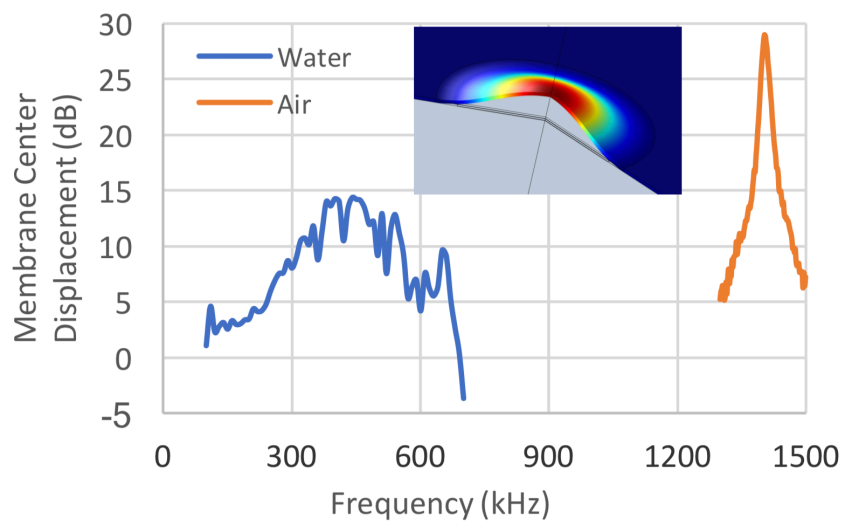

Figure 3: DHM membrane center displacement frequency response measurements of first mode of vibration in air and in water. Inset: Comsol Multiphysics Finite Element Modeling 3D simulation of PMUT membrane displacement.

\section{COMMUNICATION SYSTEM ARCHITECTURE}

The frequency response of the PMUT arrays presented a large operating bandwidth that makes them suitable for high data rate ultrasonic intra-body communications. To that end, a generic ultrasonic intra-body communication architecture, showcased in Fig. 1 , is interfaced with a PMUT array. The system architecture consists of four modules. The main module includes a processor (e.g., microcontroller (MCU)) and an FPGA that implement the physical layer and data link layer of the communication protocol stack. The communication module manages the digital-to-analog conversion (DAC) and pre-amplification of the transmitted signals, and the amplification and analog-to-digital conversion (ADC) of the received data. The system is energized through the powering module including an energy storage, e.g. a battery, and can have a sensor/actuator module to interact with the human body.

The main module of the system leverages a custom zero-padded OFDM (ZP-OFDM) transmission scheme. OFDM is a digital multi-carrier transmission scheme, which has been used extensively in underwater acoustic (UW-A) communications $[7,8,9,10]$ as it shows robustness against frequency-selective channels with long delay spreads. Given the fact that the human body is composed (up to $65 \%$ ) of water, intra-body acoustic channels show similar characteristics to UW-A channels. Therefore, OFDM stands out as a favorable transmission scheme for establishing robust high data rate communication links. In the system, to achieve energy efficiency, a zero-padding scheme is chosen over cyclic prefixing (CP), where each OFDM symbol is trailed by padded zeros. Specifically, each transmission packet is formed by $N$ ZP-OFDM symbols preceded by a preamble packet based on a pseudo-random noise (PN) sequence, which is used for packet detection and coarse time synchronization. Each ZP-OFDM symbol has $K$ subcarriers. Each subcarrier is designated as a data, pilot, or null subcarrier. Data subcarriers carry modulated information bits with different gray-coded modulation schemes (i.e. Binary-Phase-Shift-Keying (BPSK) and Quadrature-Phase-Shift-Keying (QPSK)). Pilot subcarriers are allocated to pilot symbols that are leveraged to perform channel estimation and symbol-level (fine) synchronization, while null subcarriers are used for Doppler scale estimation.

\section{EXPERIMENTAL EVALUATION}

In this section, practical implementation and performance evaluation of the aforementioned communication system model are reported.

\section{Setup}

The experimental setup consists of two ultrasonic nodes featuring the system architecture as explained in the previous section. Particularly, the ultrasonic nodes are implemented adopting USRP N210, a commercially available software-defined radio (SDR) platform based on Field Programmable Gate Arrays (FPGAs) $[11,12]$. At the transmitter side, a COTS power amplifier (PA), Mini-Circuits LZY $-22+$ with the capability of providing a gain of $43 \mathrm{~dB}$ for the operating frequency of $0.1-200 \mathrm{MHz}$, is used to amplify the $2 \mathrm{~mW}$ output power of the USRP. At the receiver side, a COTS voltage preamplifier (PreA), Teledyne RESON VP2000, which provides low-noise performance, is leveraged to amplify the received signal before feeding into the USRP.

Both transmitter and receiver chains are connected to $20 \times 20$ PMUT arrays (which were wire-bonded to printed circuit boards (PCBs) for electrical connection and for making the structure more mechanically robust) for achieving two-way conversion between electrical and ultrasonic energy. To emulate the intra-body ultrasonic channel characteristics, excepting reflections on hard elements such as bones or organs, a tissue phantom is used where PMUT arrays make contact to its surface on each side as illustrated in the Fig. 6. A coupling gel is used at the interface of the transducers and the phantom to ensure good contact and to minimize the acoustic impedance mismatch due to different materials. A demonstration video showcasing the experimental setup and real-time video steaming experiment is available at [13].

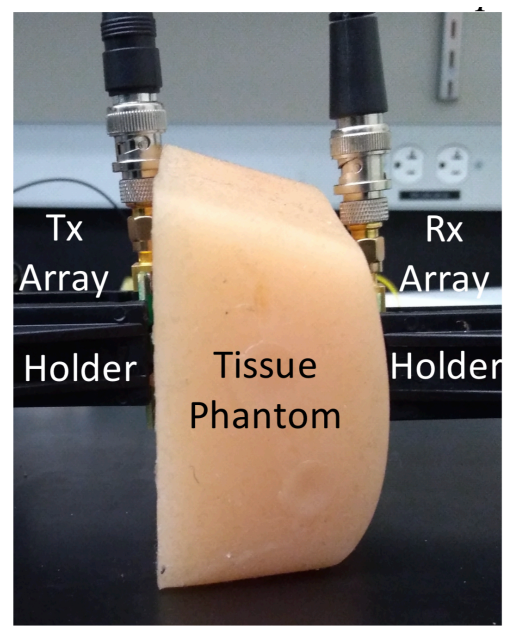

Figure 4: Experimental setup. 


\section{Results}

In this section, results from two sets of experiments are presented. The experiments are mainly concentrated on comparing the PMUTs with miniaturized ultrasonic bulk Lead Zirconate Titanate (PZT) transducers in terms of bandwidth and data rate capabilities.

In the first set of experiments, a channel estimation for both the PMUT-PMUT link and the PZT-PZT link is performed to characterize the frequency response of the communication link. Fig. 5 shows the obtained curves where the PZT response is centered around the resonant frequency with a much narrower bandwidth compared to the PMUT, which has a larger normalized amplitude over a broader spectrum. This result proves that PMUTs offer more spectrum resources compared PZTs, which could eventually lead to larger data rates.

In the second set of experiments, a ZP-OFDM scheme with a bandwidth of $B=500 \mathrm{kHz}$ is realized to quantitatively characterize and compare the data transmission capabilities of both PMUTs and PZTs. Fig. 6 shows the BER performance of PMUTs and PZTs versus SNR for different modulation schemes (i.e. BPSK and QPSK). Both PMUTs and PZTs achieved data rates of $298 \mathrm{kbit} / \mathrm{s}$ and $596 \mathrm{kbit} / \mathrm{s}$ for BPSK and QPSK, respectively. While data rates are same, for both modulation schemes, PMUTs outperform PZTs in terms of BER performance. Given that the same transmission bandwidth is used in both devices, this result proves the fact that PMUTs offering a more flat frequency response even in the non-resonating region could enable more spectrum resources and accordingly better performance.
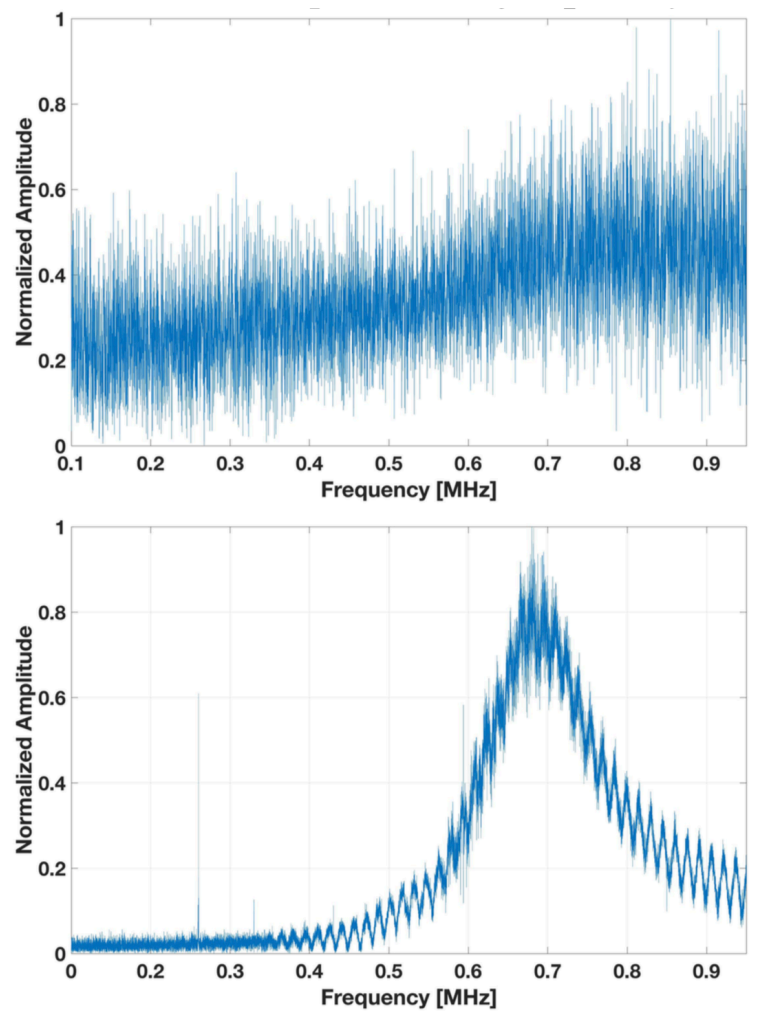

Figure 5: Channel estimation. Top: PMUT link; relatively small normalized amplitude response over frequency. Bottom: PZT transducer; narrow band around center frequency. Normalized amplitude vs frequency $(0.1-1 \mathrm{MHz})$.

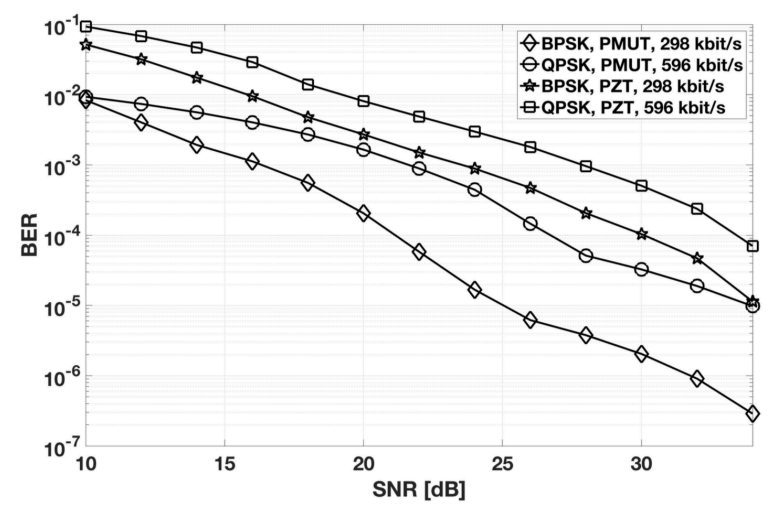

Figure 6: BER vs. SNR curves for Binary Phase shift keying (BPSK) and Quadrature Phase Shift Keying (QPSK) digital modulation schemes. $500 \mathrm{kHz}$ bandwidth. Center frequency: $700 \mathrm{kHz}$ for PZT and $600 \mathrm{kHz}$ for PMUT.

\section{CONCLUSIONS}

The present article demonstrated the feasibility of high data rate ultrasonic communications for intra-body networks by leveraging PMUTs. Specifically, it showcased successful real-time video streaming through a $5 \mathrm{~cm}$ thick tissue phantom by using $20 \times 20$ PMUT arrays both for transmission and reception.

In addition, this article presented a quantitative comparison between PZTs and PMUTs. Particularly, frequency responses of both the PMUT-PMUT link and the PZT-PZT link were measured and proved that PMUTs can offer more spectrum resources compared to PZTs. Moreover, a ZP-OFDM scheme with a bandwidth of $B=500 \mathrm{kHz}$ was tested on both the PMUT and PZT links to quantitatively characterize their data transmission capabilities. It was proved that PMUTs have better BER performance for both BPSK and QPSK modulation schemes compared to PZTs. The maximum achieved data rate with PMUTs was $0.6 \mathrm{Mbit} / \mathrm{s}$ with BER of $2 \times 10^{-6}$, which can pave the way to realize novel intra-body applications requiring high data rates.

\section{REFERENCES}

[1] Y. Lu, A. Heidari, and D. A. Horsley, "A high fill-factor annular array of high frequency piezoelectric micromachined ultrasonic transducers," Journal of Microelectromechanical Systems, vol. 24, no. 4, pp. 904-913, 2015.

[2] Y. Lu, H. Tang, S. Fung, Q. Wang, J. M. Tsai, M. Daneman, B. E. Boser, and D. A. Horsley, "Ultrasonic fingerprint sensor using a piezoelectric micromachined ultrasonic transducer array integrated with complementary metal oxide semiconductor electronics," Applied Physics Letters, vol. 106, no. 26, p. 263503, 2015.

[3] R. J. Przybyla, H. Y. Tang, A. Guedes, S. E. Shelton, D. A. Horsley, and B. E. Boser, "3d ultrasonic rangefinder on a chip," IEEE Journal of Solid-State Circuits, vol. 50, no. 1, pp. 320-334, 2015.

[4] Y. Lu, O. Rozen, H.-Y. Tang, G. L. Smith, S. Fung, B. E. Boser, R. G. Polcawich, and D. A. Horsley, "Broadband piezoelectric micromachined ultrasonic transducers based on dual resonance modes," in Micro Electro Mechanical Systems (MEMS), 2015 28th IEEE International Conference on, pp. 146-149, IEEE, 2015. 
[5] A. Koulaouzidis, D. K. Iakovidis, A. Karargyris, and E. Rondonotti, "Wireless endoscopy in 2020: Will it still be a capsule?," World journal of gastroenterology: WJG, vol. 21, no. 17 , p. $5119,2015$.

[6] G. E. Santagati and T. Melodia, "Experimental evaluation of impulsive ultrasonic intra-body communications for implantable biomedical devices," IEEE Transactions on Mobile Computing, vol. 16, no. 2, pp. 367-380, 2017.

[7] S. Zhou and Z.-H. Wang, OFDM for Underwater Acoustic Communications. John Wiley and Sons, Inc., 2014.

[8] A. Radosevic, R. Ahmed, T. Duman, J. Proakis, and M. Stojanovic, "Adaptive OFDM modulation for underwater acoustic communications: Design considerations and experimental results," IEEE Journal of Oceanic Engineering, vol. 39, pp. 357-370, April 2014.

[9] E. Demirors, J. Shi, R. Guida and T. Melodia, "SEANet G2: A Toward a High-Data-Rate Software-Defined Underwater Acoustic Networking Platform," in Proc. of ACM Intl. Conf. on Underwater Networks \& Systems (WUWNet), (Shanghai, China), October 2016.

[10] E. Demirors, G. Sklivanitis, G. E. Santagati, T. Melodia, and S. N. Batalama, "A high-rate software-defined underwater acoustic modem with real-time adaptation capabilities," IEEE Access, vol. PP, no. 99, pp. 1-1, 2018.

[11] E. Demirors, G. Sklivanitis, T. Melodia, S. N. Batalama, and D. A. Pados, "Software-defined underwater acoustic networks: toward a high-rate real-time reconfigurable modem," IEEE Communications Magazine, vol. 53, pp. 64-71, November 2015.

[12] E. Demirors, G. Alba, G. E. Santagati, and T. Melodia, "High Data Rate Ultrasonic Communications for Wireless Intra-Body Networks," in Proc. of IEEE Symposium on Local and Metropolitan Area Networks (LANMAN), (Rome, Italy), June 2016.

[13] E. Demirors, R. Guida, and B. Herrera, "PMUT communications." http://www.ece.neu.edu/wineslab/ pmut_video.php. 